\title{
Formulae for the Generalized Drazin Inverse of a Block Matrix in Banach Algebras
}

\author{
Xiaoji Liu and Xiaolan Qin \\ Faculty of Science, Guangxi University for Nationalities, Nanning 530006, China \\ Correspondence should be addressed to Xiaoji Liu; xiaojiliu72@126.com \\ Received 15 June 2015; Accepted 1 October 2015 \\ Academic Editor: Seppo Hassi
}

Copyright ( 2015 X. Liu and X. Qin. This is an open access article distributed under the Creative Commons Attribution License, which permits unrestricted use, distribution, and reproduction in any medium, provided the original work is properly cited.

We present some new representations for the generalized Drazin inverse of a block matrix in a Banach algebra under conditions weaker than those used in resent papers on the subject.

\section{Introduction}

The Drazin inverse has applications in a number of areas such as control theory, Markov chains, singular differential and difference equations, and iterative methods in numerical linear algebra. Representations for the Drazin inverse of block matrices under certain conditions were given in the literature [1-5]. Generalized inverses of block matrices have important applications in automatics, probability, statistics, mathematical programming, numerical analysis, game theory, econometrics, control, and so on [6, 7]. In 1979, Campbell and Meyer proposed the problem of finding a formula for the Drazin inverse of a $2 \times 2$ matrix in terms of its various blocks, where the blocks on the diagonal are required to be square matrices [7]. At the present time, there is no known complete solution to this problem.

In this paper, we present the representation for the Drazin inverse of $a+b$ under the conditions that $a^{k} b=a b$ and $b a^{\pi}=b$ and that $a^{k} b=a b$ and $b a^{2}=a b$ (cf. Theorem 4). And we also give several representations for the Drazin inverse of $a+b$ under some weaker conditions.

Let $\mathscr{A}$ be a complex Banach algebra with the unit 1 . The sets of all Drazin invertible and quasinilpotent elements $(\sigma(a)=\{0\})$ of $\mathscr{A}$ will be denoted by $\mathscr{A}^{d}$ and $\mathscr{A}^{\text {qnil }}$, respectively.

The generalized Drazin inverse of $a \in \mathscr{A}$ (introduced by Koliha in [8]) is the element $x \in \mathscr{A}$ which satisfies

$$
\begin{aligned}
x a x & =x, \\
a x & =x a,
\end{aligned}
$$

$$
a-a^{2} x \in \mathscr{A}^{\text {qnil }}
$$

If there exist the generalized Drazin inverse, then the generalized Drazin inverse of $a$ is unique and is denoted by $a^{d}$.

Let $a \in \mathscr{A}$ and let $p \in \mathscr{A}$ be an idempotent $\left(p=p^{2}\right)$. We denote $\bar{p}=\mathbb{1}-p$. Then we can write

$$
a=p a p+p a \bar{p}+\bar{p} a p+\bar{p} a \bar{p} .
$$

Every idempotent $p \in \mathscr{A}$ induces a representation of an arbitrary element $a \in \mathscr{A}$ given by the following matrix:

$$
a=\left[\begin{array}{ll}
p a p & p a \bar{p} \\
\bar{p} a p & \bar{p} a \bar{p}
\end{array}\right]_{p} .
$$

Let $a \in \mathscr{A}^{d}$ and $a^{\pi}=\mathbb{1}-a a^{d}$ be the spectral idempotent of $a$ corresponding to $\{0\}$. It is well known that $a \in \mathscr{A}$ can be represented in the following matrix form $[9, \mathrm{Ch} .2]$ :

$$
a=\left[\begin{array}{cc}
a_{1} & 0 \\
0 & a_{2}
\end{array}\right]_{p}
$$

where $p=a a^{d}, a_{1}$ is invertible in the algebra $p \mathscr{A} p, a^{d}$ is its inverse in $p \mathscr{A} p$, and $a_{2}$ is quasinilpotent in the algebra $\bar{p} \mathscr{A} \bar{p}$. Thus, the generalized Drazin inverse of $a$ can be expressed as

$$
a^{d}=\left[\begin{array}{ll}
a_{1}^{d} & 0 \\
0 & 0
\end{array}\right]_{p} .
$$


Obviously, if $a \in \mathscr{A}^{\text {qnil }}$, then $a$ is generalized Drazin invertible and $a^{d}=0$.

\section{Preliminary Results}

First we start the following result which is proved in [10] for matrices, extended in [2] for a bounded linear operator and in [11] for arbitrary elements in a Banach algebra.

Lemma 1 (see [11, Theorem 2.3]). Let $\mathscr{A}$ be a Banach algebra, $x, y \in \mathscr{A}$, and $p \in \mathscr{A}$ an idempotent. Assume that $x$ and $y$ are represented as

$$
\begin{aligned}
& x=\left[\begin{array}{ll}
a & 0 \\
c & b
\end{array}\right]_{p}, \\
& y=\left[\begin{array}{ll}
b & c \\
0 & a
\end{array}\right]_{p} .
\end{aligned}
$$

(i) If $a \in(p \mathscr{A} p)^{d}$ and $b \in(\bar{p} \mathscr{A} \bar{p})^{d}$, then $x$ and $y$ are generalized Drazin invertible, and

$$
\begin{aligned}
& x^{d}=\left[\begin{array}{ll}
a^{d} & 0 \\
u & b^{d}
\end{array}\right]_{p}, \\
& y^{d}=\left[\begin{array}{ll}
b^{d} & u \\
0 & a^{d}
\end{array}\right]_{p},
\end{aligned}
$$

where

$$
u=\sum_{n=0}^{\infty}\left(b^{d}\right)^{n+2} c a^{n} a^{\pi}+\sum_{n=0}^{\infty} b^{\pi} b^{n} c\left(a^{d}\right)^{n+2}-b^{d} c a^{d} .
$$

(ii) If $x \in \mathscr{A}^{d}$ and $a \in(p \mathscr{A} p)^{d}$, then $b \in(\bar{p} \mathscr{A} \bar{p})^{d}$, and $x^{d}$ and $y^{d}$ are given by (7) and (8).

Lemma 2. Let $b \in \mathscr{A}^{d}, a \in \mathscr{A}^{q n i l}$.

(1) [11, Corollary 3.4] If $a b=0$, then $a+b \in \mathscr{A}^{d}$ and

$$
(a+b)^{d}=\sum_{n=0}^{\infty}\left(b^{d}\right)^{n+1} a^{n} .
$$

(2) [12, Theorem 2.2] If $b a=0$, then $a+b \in \mathscr{A}^{d}$ and

$$
(a+b)^{d}=\sum_{n=0}^{\infty} a^{n}\left(b^{d}\right)^{n+1} .
$$

Lemma 3. Let $\mathscr{A}$ be a Banach algebra and let $a \in \mathscr{A}^{d}$ have the representation (4), where $p=a a^{d}$. If $x \in p \mathscr{A}$ and $a_{1} x=b$ for some $b \in \mathscr{A}$, then $x=a^{d} b$ (in particular, if $a_{1} x=0$, then $x=0)$. If $y \in \mathscr{A} p$ and $y a_{1}=c$ for some $c \in \mathscr{A}$, then $y=c a^{d}$ (in particular, if $y a_{1}=0$, then $y=0$ ).

Proof. From $a \in \mathscr{A}^{d}$ we get $a_{1} \in \mathscr{A}^{d}, a_{1}^{d}=a^{d}$, and $a a^{d}=a_{1} a_{1}^{d}$. There exists $u \in \mathscr{A}$ such that $x=p u$. Since $b=a_{1} x$, we obtain $a^{d} b=a^{d} a_{1} x=a a^{d} x=p x=p p u=p u=x$. The proof for $y$ is similar.

\section{Main Results}

In [13, Theorem 3.2] authors gave an explicit representation for $(a+b)^{d}$ under conditions $a^{\pi} b=b$ and $a^{2} b a^{\pi}=a b^{2} a^{\pi}=0$. Here we replace the last condition by the condition $a^{k} b=a b$; we will get a much simpler expression for $(a+b)^{d}$.

Theorem 4. Let $\mathscr{A}$ be a Banach algebra and let $a, b \in \mathscr{A}^{d}$. If there exist $k \in \mathbb{N}, k>1$, such that $a^{k} b=a b$ and $b a^{\pi}=b$, then $a+b \in \mathscr{A}^{d}$ and

$$
\begin{aligned}
(a+b)^{d} & \\
= & a^{d}+a^{\pi} \sum_{n=0}^{\infty}\left(b^{d}\right)^{n+1} a^{n}-a^{d} b \sum_{n=0}^{\infty}\left(b^{d}\right)^{n+1} a^{n} \\
& +\sum_{n=0}^{\infty}\left(a^{d}\right)^{n+2} b(a+b)^{n} b^{\pi} \\
& -\sum_{n=0}^{\infty} \sum_{k=0}^{\infty}\left(a^{d}\right)^{n+2} b(a+b)^{n}\left(b^{d}\right)^{k+1} a^{k+1}, \\
\|(a+ & b)^{d}-a^{d} \| \\
\leq & {\left[\left\|a^{\pi}\right\|+\left\|a^{d}\right\|\|b\|\right] \sum_{n=0}^{\infty}\left\|b^{d}\right\|^{n+1}\left\|a^{n}\right\| } \\
& +\sum_{n=0}^{\infty}\left\|a^{d}\right\|^{n+2}\|b\|\|a+b\|^{n}\left\|b^{\pi}\right\| \\
& +\sum_{n=0}^{\infty} \sum_{k=0}^{\infty}\left\|a^{d}\right\|^{n+2}\|b\|\|a+b\|^{n}\left[\left\|b^{d}\right\|^{k+1}\|a\|^{k+1}\right] .
\end{aligned}
$$

Proof. Let $p=a a^{d}$. We can represent $a$ as in (4), where $a_{1}$ is invertible in the subalgebra $p \mathscr{A} p$ and $a_{2}$ is quasinilpotent. Hence,

$$
a^{d}=\left[\begin{array}{ll}
a_{1}^{d} & 0 \\
0 & 0
\end{array}\right]_{p}
$$

Let us write

$$
b=\left[\begin{array}{ll}
b_{1} & b_{2} \\
b_{3} & b_{4}
\end{array}\right]
$$

From $b a^{\pi}=b$ and

$$
b\left(\mathbb{1}-a^{\pi}\right)=\left[\begin{array}{ll}
b_{1} & 0 \\
b_{3} & 0
\end{array}\right]_{p}=\left[\begin{array}{ll}
0 & 0 \\
0 & 0
\end{array}\right]_{p},
$$

we get $b_{1}=b_{3}=0$. Thus, $b$ and $a+b$ can be represented as

$$
\begin{aligned}
b & =\left[\begin{array}{ll}
0 & b_{2} \\
0 & b_{4}
\end{array}\right]_{p}, \\
a+b & =\left[\begin{array}{ll}
a_{1} & b_{2} \\
0 & a_{2}+b_{4}
\end{array}\right]_{p} .
\end{aligned}
$$


By Lemma 1 and $b \in \mathscr{A}^{d}$, we get $b_{4} \in(\bar{p} \mathscr{A} \bar{p})^{d}$ and

$$
b^{d}=\left[\begin{array}{cc}
0 & b_{2}\left(b_{4}^{d}\right)^{2} \\
0 & b_{4}^{d}
\end{array}\right]_{p} .
$$

An elementary computation from (17) and $b^{\pi}=\mathbb{1}-b b^{d}$ leads to

$$
b^{\pi}=\left[\begin{array}{cc}
p & -b_{2} b_{4}^{d} \\
0 & b_{4}^{\pi}
\end{array}\right]_{p} .
$$

Since $a^{k} b=a b$,

$$
\begin{gathered}
a^{k} b=\left[\begin{array}{ll}
0 & a_{1}^{k} b_{2} \\
0 & a_{2}^{k} b_{4}
\end{array}\right]_{p}, \\
a b=\left[\begin{array}{ll}
0 & a_{1} b_{2} \\
0 & a_{2} b_{4}
\end{array}\right]_{p},
\end{gathered}
$$

we get $a_{2}^{k} b_{4}=a_{2} b_{4}$. An induction argument proves $a_{2}^{r(k-1)+1} b_{4}=a_{2} b_{4}$ for any $r \in \mathbb{N}$. Let us denote $m_{r}=r(k-1)+1$ and observe that since $k>1$, then $\left\{a_{2}^{m_{r}}\right\}_{r=1}^{\infty}$ is a subsequence of $\left\{a_{2}^{r}\right\}_{r=1}^{\infty}$. Since $a_{2}$ is quasinilpotent, we get

$$
\begin{aligned}
\left\|a_{2} b_{4}\right\|^{1 / m_{r}} & =\left\|a_{2}^{m_{r}} b_{4}\right\|^{1 / m_{r}} \leq\left\|a_{2}^{m_{r}}\right\|^{1 / m_{r}}\left\|b_{4}\right\|^{1 / m_{r}} \\
& \stackrel{r \rightarrow \infty}{\longrightarrow} 0 .
\end{aligned}
$$

Hence $a_{2} b_{4}=0$. By Lemma 2, we have that $a_{2}+b_{4} \in \mathscr{A}^{d}$, because $b_{4} \in \mathscr{A}^{d}, a_{2} \in \mathscr{A}^{\text {qnil }}$, and $a_{2} b_{4}=0$. Also, Lemma 2 allows us to obtain

$$
\left(a_{2}+b_{4}\right)^{d}=\sum_{n=0}^{\infty}\left(b_{4}^{d}\right)^{n+1} a_{2}^{n} .
$$

Again, from Lemma 1 and (16), we get that $a+b \in \mathscr{A}^{d}$ and

$$
(a+b)^{d}=\left[\begin{array}{cc}
a_{1}^{d} & u \\
0 & \left(a_{2}+b_{4}\right)^{d}
\end{array}\right],
$$

where

$$
\begin{aligned}
u= & \sum_{n=0}^{\infty}\left(a_{1}^{d}\right)^{n+2} b_{2}\left(a_{2}+b_{4}\right)^{n}\left(a_{2}+b_{4}\right)^{\pi} \\
& +\sum_{n=0}^{\infty} a_{1}^{\pi} a_{1}^{n} b_{2}\left[\left(a_{2}+b_{4}\right)^{d}\right]^{n+2}-a_{1}^{d} b_{2}\left(a_{2}+b_{4}\right)^{d} .
\end{aligned}
$$

Observe that since $a_{1} \in(p \mathscr{A} p)^{-1}$, then $a_{1}^{\pi}=0$. Hence, the expression of $u$ reduces to

$$
\begin{aligned}
u= & \sum_{n=0}^{\infty}\left(a_{1}^{d}\right)^{n+2} b_{2}\left(a_{2}+b_{4}\right)^{n}\left(a_{2}+b_{4}\right)^{\pi} \\
& -a_{1}^{d} b_{2}\left(a_{2}+b_{4}\right)^{d} .
\end{aligned}
$$

From $a_{2} b_{4}=0$ we get $a_{2} b_{4}^{d}=a_{2} b_{4}\left(b_{4}^{d}\right)^{2}=0$. Hence

$$
\begin{aligned}
\left(a_{2}+b_{4}\right)^{\pi}= & \bar{p}-\left(a_{2}+b_{4}\right)\left(a_{2}+b_{4}\right)^{d} \\
= & \bar{p}-\left(a_{2}+b_{4}\right) \sum_{n=0}^{\infty}\left(b_{4}^{d}\right)^{n+1} a_{2}^{n} \\
= & \bar{p}-b_{4}\left(b_{4}^{d}+\left(b_{4}^{d}\right)^{2} a_{2}+\left(b_{4}^{d}\right)^{3} a_{2}^{2}+\cdots\right) \\
= & \bar{p}-b_{4} b_{4}^{d} \\
& -\left(b_{4}\left(b_{4}^{d}\right)^{2} a_{2}+b_{4}\left(b_{4}^{d}\right)^{3} a_{2}^{2}+\cdots\right) \\
= & b_{4}^{\pi}-\left(b_{4}^{d} a_{2}+\left(b_{4}^{d}\right)^{2} a_{2}^{2}+\cdots\right) .
\end{aligned}
$$

So, we get

$$
\begin{aligned}
u= & \sum_{n=0}^{\infty}\left(a_{1}^{d}\right)^{n+2} b_{2}\left(a_{2}+b_{4}\right)^{n} b_{4}^{\pi} \\
& -\sum_{n=0}^{\infty} \sum_{k=0}^{\infty}\left(a_{1}^{d}\right)^{n+2} b_{2}\left(a_{2}+b_{4}\right)^{n}\left(b_{4}^{d}\right)^{k+1} a_{2}^{k+1} \\
& -a_{1}^{d} b_{2} \sum_{n=0}^{\infty}\left(b_{4}^{d}\right)^{n+1} a_{2}^{n} .
\end{aligned}
$$

Observe that (22) and $a_{1}^{d}=a^{d}$ yield

$$
(a+b)^{d}=a^{d}+\left(a_{2}+b_{4}\right)^{d}+u \text {. }
$$

We will express $\left(a_{2}+b_{4}\right)^{d}$ using (21) in terms only of $a$ and $b$. We have from (17)

$$
\begin{aligned}
& a^{\pi}\left(b^{d}\right)^{n+1} a^{n}=\left[\begin{array}{ll}
0 & 0 \\
0 & \bar{p}
\end{array}\right]_{p}\left[\begin{array}{cc}
0 & b_{2}\left(b_{4}^{d}\right)^{n+2} \\
0 & \left(b_{4}^{d}\right)^{n+1}
\end{array}\right]_{p}\left[\begin{array}{cc}
a_{1}^{n} & 0 \\
0 & a_{2}^{n}
\end{array}\right]_{p} \\
& =\left[\begin{array}{ccc}
0 & 0 \\
0 & \left(b_{4}^{d}\right)^{n+1} & a_{2}^{n}
\end{array}\right]_{p}=\left(b_{4}^{d}\right)^{n+1} a_{2}^{n} .
\end{aligned}
$$

Hence from (21) we obtain

$$
\left(a_{2}+b_{4}\right)^{d}=a^{\pi} \sum_{n=0}^{\infty}\left(b^{d}\right)^{n+1} a^{n} .
$$


Also we have (we have written with an asterisk any entry whose exactly expression is not necessary)

$$
\begin{aligned}
& \left(a^{d}\right)^{n+2} b(a+b)^{n} b^{\pi}=\left[\begin{array}{rr}
\left(a_{1}^{d}\right)^{n+2} & 0 \\
0 & 0
\end{array}\right]_{p}\left[\begin{array}{ll}
0 & b_{2} \\
0 & b_{4}
\end{array}\right]_{p} \\
& \cdot\left[\begin{array}{cc}
a_{1}^{n} & * \\
0 & \left(a_{2}+b_{4}\right)^{n}
\end{array}\right]_{p}\left[\begin{array}{cc}
p & -b_{2} b_{4}^{d} \\
0 & b_{4}^{\pi}
\end{array}\right]_{p} \\
& =\left[\begin{array}{cc}
0 & \left(a_{1}^{d}\right)^{n+2} b_{2}\left(a_{2}+b_{4}\right)^{n} b_{4}^{\pi} \\
0 & 0
\end{array}\right]_{p}=\left(a_{1}^{d}\right)^{n+2} \\
& \cdot b_{2}\left(a_{2}+b_{4}\right)^{n} b_{4}^{\pi} \text {, } \\
& a^{d} b\left(b^{d}\right)^{n+1} a^{n}=\left[\begin{array}{cc}
a_{1}^{d} & 0 \\
0 & 0
\end{array}\right]_{p}\left[\begin{array}{ll}
0 & b_{2} \\
0 & b_{4}
\end{array}\right]_{p}\left[\begin{array}{cc}
0 & * \\
0 & \left(b_{4}^{d}\right)^{n+1}
\end{array}\right]_{p} \\
& \cdot\left[\begin{array}{cc}
a_{1}^{n} & 0 \\
0 & a_{2}^{n}
\end{array}\right]_{p}=\left[\begin{array}{ccc}
0 & a_{1}^{d} b_{2}\left(b_{4}^{d}\right)^{n+1} & a_{2}^{n} \\
0 & 0
\end{array}\right]_{p} \\
& =a_{1}^{d} b_{2}\left(b_{4}^{d}\right)^{n+1} a_{2}^{n}, \\
& \left(a_{1}^{d}\right)^{n+2} b(a+b)^{n}\left(b^{d}\right)^{k+1} a^{k+1}=\left[\begin{array}{rr}
\left(a_{1}^{d}\right)^{n+2} & 0 \\
0 & 0
\end{array}\right]_{p} \\
& \cdot\left[\begin{array}{ll}
0 & b_{2} \\
0 & b_{4}
\end{array}\right]_{p}\left[\begin{array}{cc}
a_{1}^{n} & * \\
0 & \left(a_{2}+b_{4}\right)^{n}
\end{array}\right]_{p}\left[\begin{array}{cc}
0 & * \\
0 & \left(b_{4}^{d}\right)^{k+1}
\end{array}\right]_{p} \\
& \cdot\left[\begin{array}{cc}
a_{1}^{k+1} & 0 \\
0 & a_{2}^{k+1}
\end{array}\right]_{p} \\
& =\left[\begin{array}{cc}
0 & \left(a_{1}^{d}\right)^{n+2} b_{2}\left(a_{2}+b_{4}\right)^{n}\left(b_{4}^{d}\right)^{k+1} a_{2}^{k+1} \\
0 & 0
\end{array}\right]_{p} \\
& =\left(a_{1}^{d}\right)^{n+2} b_{2}\left(a_{2}+b_{4}\right)^{n}\left(b_{4}^{d}\right)^{k+1} a_{2}^{k+1} .
\end{aligned}
$$

From (27), (29), and (30), it follows (11). The inequality (12) trivially follows from (11).

If $\mathscr{A}$ is a Banach algebra, then we can define another multiplication in $\mathscr{A}$ by $a \odot b=b a$. It is trivial that $(\mathscr{A}, \odot)$ is a Banach algebra. If we apply Theorem 4 to this new algebra, we can immediately establish the following result.

Theorem 5. Let $\mathscr{A}$ be a Banach algebra and let $a, b \in \mathscr{A}^{d}$. If there exist $k \in \mathbb{N}, k>1$, such that $b a^{k}=b a$ and $a^{\pi} b=b$, then $a+b \in \mathscr{A}^{d}$ and

$$
\begin{aligned}
& (a+b)^{d} \\
& =a^{d}+\sum_{n=0}^{\infty} a^{n}\left(b^{d}\right)^{n+1} a^{\pi}-\sum_{n=0}^{\infty} a^{n}\left(b^{d}\right)^{n+1} b a^{d}
\end{aligned}
$$

$$
\begin{aligned}
& +b^{\pi} \sum_{n=0}^{\infty}(a+b)^{n} b\left(a^{d}\right)^{n+2} \\
& -\sum_{n=0}^{\infty} \sum_{k=0}^{\infty} a^{k+1}\left(b^{d}\right)^{k+1}(a+b)^{n} b\left(a^{d}\right)^{n+2}, \\
& \left\|(a+b)^{d}-a^{d}\right\| \\
& \leq \sum_{n=0}^{\infty}\left\|a^{n}\right\|\left\|b^{d}\right\|^{n+1}\left[\left\|a^{\pi}\right\|+\|b\|\left\|a^{d}\right\|\right] \\
& +\left\|b^{\pi}\right\| \sum_{n=0}^{\infty}\|a+b\|^{n}\|b\|\left\|a^{d}\right\|^{n+2} \\
& +\sum_{n=0}^{\infty}\left[\sum_{k=0}^{\infty}\|a\|^{k+1}\left\|b^{d}\right\|^{k+1}\right]\|a+b\|^{n}\|b\|\left\|a^{d}\right\|^{n+2} .
\end{aligned}
$$

Observe that we can obtain paired results as Theorems 4 and 5 . We will not write explicitly these (trivially obtained) results.

Theorem 6. Let $\mathscr{A}$ be a Banach algebra and let $a, b \in \mathscr{A}^{d}$ be such that $w=(a+b) a a^{d} \in \mathscr{A}^{d}$. If there exist $k \in \mathbb{N}, k>1$, such that $a^{k} b=a b$ and $b a=a b^{2}$, then $a+b \in \mathscr{A}^{d}$ and

$$
\begin{aligned}
(a+b)^{d}= & w^{d}+a^{\pi} b^{d}+\sum_{n=0}^{\infty}\left(w^{d}\right)^{n+2} b a^{\pi}(a+b)^{n} b^{\pi} \\
& +\sum_{n=0}^{\infty} w^{\pi} w^{n} b a^{\pi}\left(b^{d}\right)^{n+2}-w^{d} b a^{\pi} b^{d} .
\end{aligned}
$$

Proof. Let us consider the matrix representations of $a, a^{d}$, and $b$ given in (4), (13), and (14) relative to the idempotent $p=$ $a a^{d}$. As in the proof of Theorem 4 , from $a^{k} b=a b$, we get $a_{2} b_{3}=a_{2} b_{4}=0$.

From $b a=a b^{2}$ we have

$$
\begin{aligned}
a b^{2} & =\left[\begin{array}{cc}
a_{1} b_{1}^{2}+a_{1} b_{2} b_{3} & a_{1} b_{1} b_{2}+a_{1} b_{2} b_{4} \\
a_{2} b_{3} b_{1}+a_{2} b_{4} b_{3} & a_{2} b_{3} b_{2}+a_{2} b_{4}^{2}
\end{array}\right]_{p} \\
& =\left[\begin{array}{cc}
a_{1} b_{1}^{2}+a_{1} b_{2} b_{3} & a_{1} b_{1} b_{2}+a_{1} b_{2} b_{4} \\
0 & 0
\end{array}\right]_{p}, \\
b a & =\left[\begin{array}{ll}
b_{1} a_{1} & b_{2} a_{2} \\
b_{3} a_{1} & b_{4} a_{2}
\end{array}\right]_{p} .
\end{aligned}
$$

Therefore, $b_{3} a_{1}=0$ and $a_{2} b_{4}=b_{4} a_{2}=0$. From Lemma 3 we get $b_{3}=0$. Hence

$$
\begin{aligned}
b & =\left[\begin{array}{ll}
b_{1} & b_{2} \\
0 & b_{4}
\end{array}\right]_{p}, \\
a+b & =\left[\begin{array}{cc}
a_{1}+b_{1} & b_{2} \\
0 & a_{2}+b_{4}
\end{array}\right]_{p},
\end{aligned}
$$




$$
\begin{aligned}
w & =(a+b) a a^{d}=\left[\begin{array}{cc}
a_{1}+b_{1} & b_{2} \\
0 & a_{2}+b_{4}
\end{array}\right]_{p}\left[\begin{array}{ll}
p & 0 \\
0 & 0
\end{array}\right]_{p} \\
& =\left[\begin{array}{cc}
a_{1}+b_{1} & 0 \\
0 & 0
\end{array}\right]_{p}=a_{1}+b_{1} .
\end{aligned}
$$

Lemma 2 implies that $a_{2}+b_{4} \in \mathscr{A}^{d}$ because $a \in \mathscr{A}^{\text {qnil }}$, $b \in \mathscr{A}^{d}$, and $a_{2} b_{4}=0$. Also, we obtain

$$
\left(a_{2}+b_{4}\right)^{d}=\sum_{n=0}^{\infty}\left(b_{4}^{d}\right)^{n+1} a_{2}^{n} .
$$

In this situation, we obtain

$$
(a+b)^{d}=\left[\begin{array}{cc}
w^{d} & u \\
0 & \left(a_{2}+b_{4}\right)^{d}
\end{array}\right]_{p}=w^{d}+u+\left(a_{2}+b_{4}\right)^{d},
$$

$u$

$$
\begin{aligned}
= & \sum_{n=0}^{\infty}\left(\left(a_{1}+b_{1}\right)^{d}\right)^{n+2} b_{2}\left(a_{2}+b_{4}\right)^{n}\left(a_{2}+b_{4}\right)^{\pi} \\
& +\sum_{n=0}^{\infty}\left(a_{1}+b_{1}\right)^{\pi}\left(a_{1}+b_{1}\right)^{n} b_{2}\left(\left(a_{2}+b_{4}\right)^{d}\right)^{n+2} \\
& -\left(a_{1}+b_{1}\right)^{d} b_{2}\left(a_{2}+b_{4}\right)^{d} .
\end{aligned}
$$

Since $a_{2} b_{4}=b_{4} a_{2}=0$, we have $b_{4}^{d} a_{2}=\left(b_{4}^{d}\right)^{2} b_{4} a_{2}=0$. Thus (35) reduces to

$$
\left(a_{2}+b_{4}\right)^{d}=b_{4}^{d}=a^{\pi} b^{d} .
$$

From $a_{2} b_{4}=0$ we have $a_{2} b_{4}^{d}=a_{2} b_{4}\left(b_{4}^{d}\right)^{2}=0$. Therefore,

$$
\begin{aligned}
\left(a_{2}+b_{4}\right)^{\pi} & =\bar{p}-\left(a_{2}+b_{4}\right)\left(a_{2}+b_{4}\right)^{d} \\
& =\bar{p}-a_{2} b_{4}^{d}-b_{4} b_{4}^{d}=b_{4}^{\pi},
\end{aligned}
$$

so we get

$$
\begin{aligned}
u= & \sum_{n=0}^{\infty}\left(\left(a_{1}+b_{1}\right)^{d}\right)^{n+2} b_{2}\left(a_{2}+b_{4}\right)^{n} b_{4}^{\pi} \\
& +\sum_{n=0}^{\infty}\left(a_{1}+b_{1}\right)^{\pi}\left(a_{1}+b_{1}\right)^{n} b_{2}\left(b_{4}^{d}\right)^{n+2} \\
& -\left(a_{1}+b_{1}\right)^{d} b_{2} b_{4}^{d} .
\end{aligned}
$$

We have

$$
\begin{aligned}
& \left(w^{d}\right)^{n+2} b a^{\pi}(a+b)^{n} b^{\pi}=\left[\begin{array}{cc}
\left(\left(a_{1}+b_{1}\right)^{d}\right)^{n+2} & 0 \\
0 & 0
\end{array}\right]_{p} \\
& \cdot\left[\begin{array}{ll}
b_{1} & b_{2} \\
0 & b_{4}
\end{array}\right]_{p}\left[\begin{array}{ll}
0 & 0 \\
0 & \bar{p}
\end{array}\right]_{p}\left[\begin{array}{cc}
b_{1}^{n} & * \\
0 & \left(a_{2}+b_{4}\right)^{n}
\end{array}\right]_{p}\left[\begin{array}{cc}
b_{1}^{\pi} & * \\
0 & b_{4}^{\pi}
\end{array}\right]_{p} \\
& =\left[\begin{array}{ll}
0 & \left(\left(a_{1}+b_{1}\right)^{d}\right)^{n+2} b_{2}\left(a_{2}+b_{4}\right)^{n} b_{4}^{\pi} \\
0 & 0
\end{array}\right] \\
& =\left(\left(a_{1}+b_{1}\right)^{d}\right)^{n+2} b_{2}\left(a_{2}+b_{4}\right)^{n} b_{4}^{\pi} .
\end{aligned}
$$

Also,

$$
\begin{gathered}
w^{\pi} w^{n} b a^{\pi}\left(b^{d}\right)^{n+2}=\left[\begin{array}{cc}
\left(a_{1}+b_{1}\right)^{\pi} & 0 \\
0 & \bar{p}
\end{array}\right]_{p} \\
\cdot\left[\begin{array}{cc}
\left(a_{1}+b_{1}\right)^{n} & 0 \\
0 & 0
\end{array}\right]_{p}\left[\begin{array}{ll}
b_{1} & b_{2} \\
0 & b_{4}
\end{array}\right]_{p}\left[\begin{array}{ll}
0 & 0 \\
0 & \bar{p}
\end{array}\right]_{p} \\
\cdot\left[\begin{array}{cc}
\left(b_{1}^{d}\right)^{n+2} & * \\
0 & \left(b_{4}^{d}\right)^{n+2}
\end{array}\right]_{p} \\
=\left[\begin{array}{cc}
0 & \left(a_{1}+b_{1}\right)^{\pi}\left(a_{1}+b_{1}\right)^{n} b_{2}\left(b_{4}^{d}\right)^{n+2} \\
0 & 0
\end{array}\right]_{p} \\
=\left(a_{1}+b_{1}\right)^{\pi}\left(a_{1}+b_{1}\right)^{n} b_{2}\left(b_{4}^{d}\right)^{n+2}, \\
w^{d} b a^{\pi} b^{d}=\left[\begin{array}{cc}
\left(a_{1}+b_{1}\right)^{d} & 0 \\
0 & 0
\end{array}\right]_{p}\left[\begin{array}{cc}
b_{1} & b_{2} \\
0 & b_{4}
\end{array}\right]_{p}\left[\begin{array}{ll}
0 & 0 \\
0 & \bar{p}
\end{array}\right]_{p} \\
\cdot\left[\begin{array}{cc}
b_{1}^{d} & * \\
0 & b_{4}^{d}
\end{array}\right]_{p}=\left[\begin{array}{ll}
0 & \left(a_{1}+b_{1}\right)^{d} \\
0 & b_{2} b_{4}^{d} \\
0 & 0
\end{array}\right]=\left(a_{1}+b_{1}\right)^{d} \\
\cdot b_{2} b_{4}^{d} \cdot \quad
\end{gathered}
$$

From (36), (38), (41), and (42), it follows (32).

The proof is finished.

As we have commented before, we can obtain a paired result by considering the Banach algebra $\mathscr{A}$ with the product $a \odot b=b a$. The key hypothesis of this new result will be $b a^{k}=b a$ and $b^{2} a=a b$.

Theorem 7. Let $\mathscr{A}$ be a Banach algebra and let $a, b \in \mathscr{A}^{d}$ be such that $w=a a^{d}(a+b) \in \mathscr{A}^{d}$. If there exist $k \in \mathbb{N}, k>1$, such that $b a^{k}=b a$ and $b^{2} a=a b$, then $a+b \in \mathscr{A}^{d}$ and

$$
\begin{aligned}
(a+b)^{d}= & w^{d}+b^{d} a^{\pi}-b^{d} a^{\pi} b w^{d} \\
& +\sum_{n=0}^{\infty}\left(b^{d}\right)^{n+2} a^{\pi} b w^{n} w^{\pi} \\
& +b^{\pi} \sum_{n=0}^{\infty}(a+b)^{n} a^{\pi} b\left(w^{d}\right)^{n+2} .
\end{aligned}
$$


Theorem 8. Let $\mathscr{A}$ be a Banach algebra and let $a, b \in \mathscr{A}^{d}$ be such that $w=a a^{d}(a+b) \in \mathscr{A}^{d}$. If there exist $k, n, m \in \mathbb{N}$, $k>1$, such that $a^{n} b=b a^{m}$ and $a^{k} b=a b$, then $a+b \in \mathscr{A}^{d}$ and

$$
(a+b)^{d}=w^{d}+\sum_{n=0}^{\infty}\left(b^{d}\right)^{n+1} a^{n} a^{\pi} .
$$

Proof. Let us consider the matrix representations of $a, a^{d}$, and $b$ given in (4), (13), and (14) relative to the idempotent $p=$ $a a^{d}$. From $a^{n} b=b a^{m}$, we have

$$
\begin{aligned}
& a_{1}^{n} b_{1}=b_{1} a_{1}^{m}, \\
& a_{1}^{n} b_{2}=b_{2} a_{2}^{m}, \\
& a_{2}^{n} b_{3}=b_{3} a_{1}^{m}, \\
& a_{2}^{n} b_{4}=b_{4} a_{2}^{m} .
\end{aligned}
$$

The second equality of (45) implies $a_{1}^{n k} b_{2}=b_{2} a_{2}^{m k}$ for any $k \in \mathbb{N}$. By Lemma 3 we get $b_{2}=\left(a^{d}\right)^{n k} b_{2} a_{2}^{m k}$. Hence

$$
\left\|b_{2}\right\|^{1 /(m k)} \leq\left\|\left(a^{d}\right)^{n k}\right\|^{1 /(m k)}\left\|b_{2}\right\|^{1 /(m k)}\left\|a_{2}^{m k}\right\|^{1 /(m k)}
$$

Therefore, if $\beta$ denotes $\lim _{k \rightarrow \infty}\left\|b_{2}\right\|^{1 /(m k)}$ and $\alpha$ denotes $\lim _{k \rightarrow \infty}\left\|\left(a^{d}\right)^{n k}\right\|^{1 /(m k)}$, then $0 \leq \beta \leq \beta \alpha r\left(a_{2}\right)$. Recall that $a_{2}$ is quasinilpotent, and thus $r\left(a_{2}\right)=0$. We have just proved $\beta=0$ or, equivalently, $b_{2}=0$. Similarly we obtain $b_{3}=0$.

Thus, $b$ and $a+b$ can be represented as

$$
\begin{aligned}
b & =\left[\begin{array}{ll}
b_{1} & 0 \\
0 & b_{4}
\end{array}\right]_{p}, \\
a+b & =\left[\begin{array}{cc}
a_{1}+b_{1} & 0 \\
0 & a_{2}+b_{4}
\end{array}\right]_{p} .
\end{aligned}
$$

Since $a^{k} b=a b$, by the proof of Theorem 4 , we get $a_{2} b_{4}=$ 0 . From Lemma 2, we have $a_{2}+b_{4} \in \mathscr{A}^{d}$, because $b_{4} \in \mathscr{A}^{d}$, $a_{2} \in \mathscr{A}^{\text {qnil }}$, and $a_{2} b_{4}=0$. Also, Lemma 2 allows us to obtain

$$
\left(a_{2}+b_{4}\right)^{d}=\sum_{n=0}^{\infty}\left(b_{4}^{d}\right)^{n+1} a_{2}^{n} .
$$

We have $a+b \in \mathscr{A}^{d}$ if and only if $a_{1}+b_{1} \in \mathscr{A}^{d}$. But $w=a a^{d}(a+b)=a_{1}+b_{1}$. If $w \in \mathscr{A}^{d}$, then

$$
\begin{aligned}
(a+b)^{d} & =\left[\begin{array}{cc}
\left(a_{1}+b_{1}\right)^{d} & 0 \\
0 & \left(a_{2}+b_{4}\right)^{d}
\end{array}\right]_{p} \\
& =w^{d}+\sum_{n=0}^{\infty}\left(b^{d}\right)^{n+1} a^{n} a^{\pi} .
\end{aligned}
$$

The theorem is just proved.
Theorem 9. Let $\mathscr{A}$ be a Banach algebra and let $a, b \in \mathscr{A}^{d}$. If there exist $k \in \mathbb{N}, k>1$, such that $a^{k} b=a b a^{\pi}$ and $b a=a b^{2}$, then $a+b \in \mathscr{A}^{d}$ and

$$
\begin{aligned}
(a+b)^{d} & =a^{d}+a^{\pi} b^{d}+\left(a^{d}\right)^{3} b(a+b), \\
\left\|(a+b)^{d}-a^{d}\right\| & \leq\left\|a^{\pi}\right\|\left\|b^{d}\right\|+\left\|a^{d}\right\|^{3}\|b\|\|a+b\| .
\end{aligned}
$$

Proof. Let us consider the matrix representations of $a, a^{d}$, and $b$ given in (4), (13), and (14) relative to the idempotent $p=$ $a a^{d}$. Since $a^{k} b=a b a^{\pi}$,

$$
\begin{gathered}
a^{k} b=\left[\begin{array}{ll}
a_{1}^{k} b_{1} & a_{1}^{k} b_{2} \\
a_{2}^{k} b_{3} & a_{2}^{k} b_{4}
\end{array}\right]_{p}, \\
a b a^{\pi}=\left[\begin{array}{ll}
0 & a_{1} b_{2} \\
0 & a_{2} b_{4}
\end{array}\right]_{p},
\end{gathered}
$$

we get $a_{1}^{k} b_{1}=0$ and $a_{2}^{k} b_{4}=a_{2} b_{4}$; as in the proof of Theorem 4, we have $a_{2} b_{4}=0$. From Lemma 3 we get $b_{1}=0$.

From $b a=a b^{2}$ we have

$$
\begin{aligned}
a b^{2} & =\left[\begin{array}{cc}
a_{1} b_{1}^{2}+a_{1} b_{2} b_{3} & a_{1} b_{1} b_{2}+a_{1} b_{2} b_{4} \\
a_{2} b_{3} b_{1}+a_{2} b_{4} b_{3} & a_{2} b_{3} b_{2}+a_{2} b_{4}^{2}
\end{array}\right]_{p} \\
& =\left[\begin{array}{cc}
a_{1} b_{2} b_{3} & a_{1} b_{2} b_{4} \\
0 & a_{2} b_{3} b_{2}
\end{array}\right]_{p}, \\
b a & =\left[\begin{array}{ll}
b_{1} a_{1} & b_{2} a_{2} \\
b_{3} a_{1} & b_{4} a_{2}
\end{array}\right]_{p}=\left[\begin{array}{cc}
0 & b_{2} a_{2} \\
b_{3} a_{1} & b_{4} a_{2}
\end{array}\right]_{p} .
\end{aligned}
$$

Therefore, $b_{3} a_{1}=0, b_{2} a_{2}=a_{1} b_{2} b_{4}$, and $b_{4} a_{2}=a_{2} b_{3} b_{2}$. From Lemma 3 we get $b_{3}=0$ and $b_{4} a_{2}=a_{2} b_{3} b_{2}=0$. Hence

$$
\begin{gathered}
b=\left[\begin{array}{ll}
0 & b_{2} \\
0 & b_{4}
\end{array}\right]_{p}, \\
a+b=\left[\begin{array}{ll}
a_{1} & b_{2} \\
0 & a_{2}+b_{4}
\end{array}\right]_{p} .
\end{gathered}
$$

Lemma 2 implies that $a_{2}+b_{4} \in \mathscr{A}^{d}$ because $a \in \mathscr{A}^{\text {qnil }}$, $b \in \mathscr{A}^{d}$, and $a_{2} b_{4}=b_{4} a_{2}=0$; as in the proof of Theorem 6 , we obtain

$$
\begin{aligned}
& \left(a_{2}+b_{4}\right)^{d}=b_{4}^{d}, \\
& \left(a_{2}+b_{4}\right)^{\pi}=b_{4}^{\pi} .
\end{aligned}
$$


In this situation, we obtain

$$
\begin{aligned}
(a+b)^{d}= & {\left[\begin{array}{cc}
a_{1}^{d} & u \\
0 & \left(a_{2}+b_{4}\right)^{d}
\end{array}\right]_{p}=a_{1}^{d}+u+\left(a_{2}+b_{4}\right)^{d}, } \\
u= & \sum_{n=0}^{\infty}\left(a_{1}^{d}\right)^{n+2} b_{2}\left(a_{2}+b_{4}\right)^{n}\left(a_{2}+b_{4}\right)^{\pi} \\
& +\sum_{n=0}^{\infty} a_{1}^{\pi} a_{1}^{n} b_{2}\left[\left(a_{2}+b_{4}\right)^{d}\right]^{n+2} \\
& -a_{1}^{d} b_{2}\left(a_{2}+b_{4}\right)^{d} .
\end{aligned}
$$

Observe that since $a_{1} \in(p \mathscr{A} p)^{-1}$, then $a_{1}^{\pi}=0$. Hence, the expression of $u$ reduces to

$$
u=\sum_{n=0}^{\infty}\left(a_{1}^{d}\right)^{n+2} b_{2}\left(a_{2}+b_{4}\right)^{n} b_{4}^{\pi}-a_{1}^{d} b_{2} b_{4}^{d} .
$$

From $a_{2} b_{4}=b_{4} a_{2}=0$ and $b_{2} a_{2}=a_{1} b_{2} b_{4}$, we have

$$
\begin{aligned}
\left(a_{2}+b_{4}\right)^{n} & =a_{2}^{n}+b_{4}^{n}, \\
a_{1}^{d} b_{2} b_{4}^{d} & =\left(a_{1}^{d}\right)^{2} a_{1} b_{2} b_{4} b_{4}\left(b_{4}^{d}\right)^{3} \\
& =\left(a_{1}^{d}\right)^{2} b_{2} a_{2} b_{4}\left(b_{4}^{d}\right)^{2}=0 .
\end{aligned}
$$

So we get

$$
\begin{aligned}
& \left(a_{1}^{d}\right)^{n+2} b_{2}\left(a_{2}+b_{4}\right)^{n} b_{4} b_{4}^{d} \\
& =\left(a_{1}^{d}\right)^{n+2} b_{2} a_{2}^{n} b_{4} b_{4}^{d}+\left(a_{1}^{d}\right)^{n+2} b_{2} b_{4}^{n} b_{4} b_{4}^{d}=0, \\
& \left(a_{1}^{d}\right)^{n+2} b_{2}\left(a_{2}+b_{4}\right)^{n}=\left(a_{1}^{d}\right)^{n+2} b_{2} a_{2}^{n}+\left(a_{1}^{d}\right)^{n+2} b_{2} b_{4}^{n} \\
& =\left(a_{1}^{d}\right)^{n+2} b_{2} a_{2} a_{2}^{n-1}+\left(a_{1}^{d}\right)^{n+3} a_{1} b_{2} b_{4} b_{4}^{n-1} \\
& =\left(a_{1}^{d}\right)^{n+2} a_{1} b_{2} b_{4} a_{2}^{n-1}+\left(a_{1}^{d}\right)^{n+3} b_{2} a_{2} b_{4}^{n-1}=0,
\end{aligned}
$$

$n \geq 2$.

Hence

$$
\begin{aligned}
u= & \sum_{n=0}^{\infty}\left(a_{1}^{d}\right)^{n+2} b_{2}\left(a_{2}+b_{4}\right)^{n} b_{4}^{\pi}-a_{1}^{d} b_{2} b_{4}^{d} \\
= & \sum_{n=0}^{\infty}\left(a_{1}^{d}\right)^{n+2} b_{2}\left(a_{2}+b_{4}\right)^{n} \\
& -\sum_{n=0}^{\infty}\left(a_{1}^{d}\right)^{n+2} b_{2}\left(a_{2}+b_{4}\right)^{n} b_{4} b_{4}^{d} \\
= & \left(a_{1}^{d}\right)^{3} b_{2}\left(a_{2}+b_{4}\right) .
\end{aligned}
$$

Thus (55) reduces to

$$
\left(a_{2}+b_{4}\right)^{d}=b_{4}^{d}=a^{\pi} b^{d} .
$$

We have

$$
\begin{aligned}
& \left(a^{d}\right)^{3} b(a+b) \\
& \quad=\left[\begin{array}{cc}
\left(a_{1}^{d}\right)^{3} & 0 \\
0 & 0
\end{array}\right]_{p}\left[\begin{array}{ll}
0 & b_{2} \\
0 & b_{4}
\end{array}\right]_{p}\left[\begin{array}{cc}
a_{1} & b_{2} \\
0 & a_{2}+b_{4}
\end{array}\right]_{p}
\end{aligned}
$$

$$
=\left[\begin{array}{cc}
0 & \left(a_{1}^{d}\right)^{3} b_{2}\left(a_{2}+b_{4}\right) \\
0 & 0
\end{array}\right]=\left(a_{1}^{d}\right)^{3} b_{2}\left(a_{2}+b_{4}\right) .
$$

From (56), (62), and (63), it follows (50). The inequality (51) trivially follows from (50).

The proof is finished.

As we have commented before, we can obtain a paired result by considering the Banach algebra $\mathscr{A}$ with the product $a \odot b=b a$. The key hypothesis of this new result will be $b a^{k}=a^{\pi} b a$ and $b^{2} a=a b$.

Theorem 10. Let $\mathscr{A}$ be a Banach algebra and let $a, b \in \mathscr{A}^{d}$. If there exist $k \in \mathbb{N}, k>1$, such that $b a^{k}=a^{\pi} b a$ and $b^{2} a=a b$, then $a+b \in \mathscr{A}^{d}$ and

$$
\begin{aligned}
(a+b)^{d} & =a^{d}+b^{d} a^{\pi}+(a+b) b\left(a^{d}\right)^{3}, \\
\left\|(a+b)^{d}-a^{d}\right\| & \leq\left\|b^{d}\right\|\left\|a^{\pi}\right\|+\|a+b\|\|b\|\left\|a^{d}\right\|^{3} .
\end{aligned}
$$

In the rest of the paper, we will use some weaker conditions than in [11, Theorem 4.1]. For example, if we assume that $a^{k} b a^{\pi}=a b$ and $a^{\pi} b=a b^{2}$ instead of $a b a^{\pi}=0$ and $a^{\pi} b=0$, we will get a much simpler expression for $(a+b)^{d}$.

Theorem 11. Let $\mathscr{A}$ be a Banach algebra and let $a, b \in \mathscr{A}^{d}$. If there exist $k \in \mathbb{N}, k>1$, such that $a^{k} b a^{\pi}=a b$ and $a b^{2}=a^{\pi} b$, then $a+b \in \mathscr{A}^{d}$ and

$$
\begin{array}{r}
(a+b)^{d}=a^{d}+\sum_{n=0}^{\infty}\left(a^{d}\right)^{n+2} b a^{n}, \\
\left\|(a+b)^{d}-a^{d}\right\| \leq \sum_{n=0}^{\infty}\left\|a^{d}\right\|^{n+2}\|b\|\|a\|^{n} .
\end{array}
$$

Proof. Let us consider the matrix representations of $a, a^{d}$, and $b$ given in (4), (13), and (14) relative to the idempotent $p=$ $a a^{d}$. We will use the condition $a^{k} b a^{\pi}=a b$ : since

$$
\begin{aligned}
a^{k} b a^{\pi} & =\left[\begin{array}{ll}
0 & a_{1}^{k} b_{2} \\
0 & a_{2}^{k} b_{4}
\end{array}\right]_{p}, \\
a b & =\left[\begin{array}{ll}
a_{1} b_{1} & a_{1} b_{2} \\
a_{2} b_{3} & a_{2} b_{4}
\end{array}\right]_{p},
\end{aligned}
$$

we get $a_{1} b_{1}=a_{2} b_{3}=0$ and $a_{2}^{k} b_{4}=a_{2} b_{4}$; as in the proof of Theorem 4 , we have $a_{2} b_{4}=0$. From Lemma 3 we get $b_{1}=0$.

From $a b^{2}=a^{\pi} b$ we have

$$
\begin{aligned}
a b^{2} & =\left[\begin{array}{cc}
a_{1} b_{1}^{2}+a_{1} b_{2} b_{3} & a_{1} b_{1} b_{2}+a_{1} b_{2} b_{4} \\
a_{2} b_{3} b_{1}+a_{2} b_{4} b_{3} & a_{2} b_{3} b_{2}+a_{2} b_{4}^{2}
\end{array}\right]_{p} \\
& =\left[\begin{array}{cc}
a_{1} b_{2} b_{3} & a_{1} b_{2} b_{4} \\
0 & 0
\end{array}\right]_{p}, \\
a^{\pi} b & =\left[\begin{array}{ll}
0 & 0 \\
b_{3} & b_{4}
\end{array}\right]_{p} .
\end{aligned}
$$


Therefore, $b_{3}=b_{4}=0$. Hence

$$
\begin{aligned}
b & =\left[\begin{array}{ll}
0 & b_{2} \\
0 & 0
\end{array}\right]_{p}, \\
a+b & =\left[\begin{array}{ll}
a_{1} & b_{2} \\
0 & a_{2}
\end{array}\right]_{p}, \\
(a+b)^{d} & =\left[\begin{array}{ll}
a_{1}^{d} & u \\
0 & 0
\end{array}\right]_{p}=a_{1}^{d}+u,
\end{aligned}
$$

where

$$
u=\sum_{n=0}^{\infty}\left(a_{1}^{d}\right)^{n+2} b_{2} a_{2}^{n}=\sum_{n=0}^{\infty}\left(a^{d}\right)^{n+2} b a^{n} .
$$

The proof is finished.

Theorem 12. Let $\mathscr{A}$ be a Banach algebra and let $a, b \in \mathscr{A}^{d}$. If there exist $k \in \mathbb{N}, k>1$, such that $a^{k} b a^{\pi}=a b$ and $a^{\pi} b=a b a$, then $a+b \in \mathscr{A}^{d}$ and

$$
\begin{aligned}
(a+b)^{d} & =a^{d}+\left(a^{d}\right)^{2} b, \\
\left\|(a+b)^{d}-a^{d}\right\| & \leq\left\|a^{d}\right\|^{2}\|b\| .
\end{aligned}
$$

Proof. Let us consider the matrix representations of $a, a^{d}$, and $b$ given in (4), (13), and (14) relative to the idempotent $p=$ $a a^{d}$. As in the proof of Theorem 11, from $a^{k} b a^{\pi}=a b$, we get $b_{1}=0$ and $a_{2} b_{3}=a_{2} b_{4}=0$.

Since $a^{\pi} b=a b a$

$$
\begin{aligned}
a b & =\left[\begin{array}{ll}
a_{1} b_{1} & a_{1} b_{2} \\
a_{2} b_{3} & a_{2} b_{4}
\end{array}\right]_{p}=\left[\begin{array}{cc}
0 & a_{1} b_{2} \\
0 & 0
\end{array}\right]_{p}, \\
a b a & =\left[\begin{array}{cc}
0 & a_{1} b_{2} \\
0 & 0
\end{array}\right]_{p}\left[\begin{array}{cc}
a_{1} & 0 \\
0 & a_{2}
\end{array}\right]_{p}=\left[\begin{array}{cc}
0 & a_{1} b_{2} a_{2} \\
0 & 0
\end{array}\right]_{p}, \\
a^{\pi} b & =\left[\begin{array}{ll}
0 & 0 \\
b_{3} & b_{4}
\end{array}\right]_{p}
\end{aligned}
$$

we get $a_{1} b_{2} a_{2}=b_{3}=b_{4}=0$. As in the proof of Theorem 11, we have

$$
(a+b)^{d}=\left[\begin{array}{cc}
a_{1}^{d} & u \\
0 & 0
\end{array}\right]_{p}=a_{1}^{d}+u
$$

where

$$
u=\sum_{n=0}^{\infty}\left(a_{1}^{d}\right)^{n+2} b_{2} a_{2}^{n}
$$

From $a_{1} b_{2} a_{2}=0$, we get

$$
u=\sum_{n=0}^{\infty}\left(a_{1}^{d}\right)^{n+2} b_{2} a_{2}^{n}=\left(a_{1}^{d}\right)^{2} b_{2}=\left(a^{d}\right)^{2} b .
$$

The proof is finished.

\section{Conflict of Interests}

The authors declare that there is no conflict of interests regarding the publication of this paper.

\section{Acknowledgments}

This work was supported by the National Natural Science Foundation of China (no. 11361009), the Guangxi Provincial Natural Science Foundation of China (no. 2013GXNSFAA019008), and Science Research Project 2013 of the ChinaASEAN Study Center (Guangxi Science Experiment Center) of Guangxi University for Nationalities. The authors would like to thank the referees and the editors for their suitable comments.

\section{References}

[1] C. Y. Deng, "A note on the Drazin inverses with BanachiewiczSchur forms," Applied Mathematics and Computation, vol. 213, no. 1, pp. 230-234, 2009.

[2] D. S. Djordjević and P. S. Stanimirović, "On the generalized Drazin inverse and generalized resolvent," Czechoslovak Mathematical Journal, vol. 51, no. 3, pp. 617-634, 2001.

[3] D. S. Djordjević-Ilić and Y. Wei, "Representations for the Drazin inverse of bounded operators on Banach space," Electronic Journal of Linear Algebra, vol. 18, no. 1, pp. 613-627, 2009.

[4] N. Castro-González, E. Dopazo, and J. Robles, "Formulas for the Drazin inverse of special block matrices," Applied Mathematics and Computation, vol. 174, no. 1, pp. 252-270, 2006.

[5] R. Hartwig, X. Li, and Y. Wei, "Representations for the Drazin inverse of a $2 \times 2$ block matrix," SIAM Journal on Matrix Analysis and Applications, vol. 27, no. 3, pp. 757-771, 2005.

[6] S. L. Campbell, Singular Systems of Differential Equations, Pitman, San Francisco, Calif, USA, 1980.

[7] S. L. Campbell and C. D. Meyer, Generalized Inverses of Linear Transformations, Pitman, London, UK, 1979.

[8] J. J. Koliha, "A generalized drazin inverse," Glasgow Mathematical Journal, vol. 38, no. 3, pp. 367-381, 1996.

[9] D. Djordjević and V. Rakočević, Lectures on Generalized Inverses, University of Niš, Niš, Serbia, 2008.

[10] C. D. Meyer Jr. and N. J. Rose, "The index and the Drazin inverse of block triangular matrices," SIAM Journal on Applied Mathematics, vol. 33, no. 1, pp. 1-7, 1977.

[11] N. Castro-González and J. J. Koliha, "New additive results for the Drazin inverse," Proceedings of the Royal Society of Edinburgh: Section A, vol. 134, pp. 1085-1097, 2004.

[12] D. S. Djordjević and Y. Wei, "Additive results for the generalized Drazin inverse," Journal of the Australian Mathematical Society, vol. 73, no. 1, pp. 115-125, 2002.

[13] N. Castro-González and M. F. Martínez-Serrano, "Expressions for the g-Drazin inverse of additive perturbed elements in a Banach algebra," Linear Algebra and Its Applications, vol. 432, no. 8, pp. 1885-1895, 2010. 


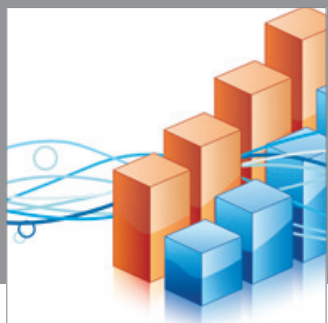

Advances in

Operations Research

mansans

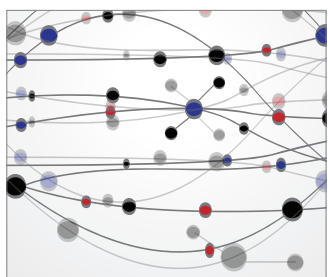

The Scientific World Journal
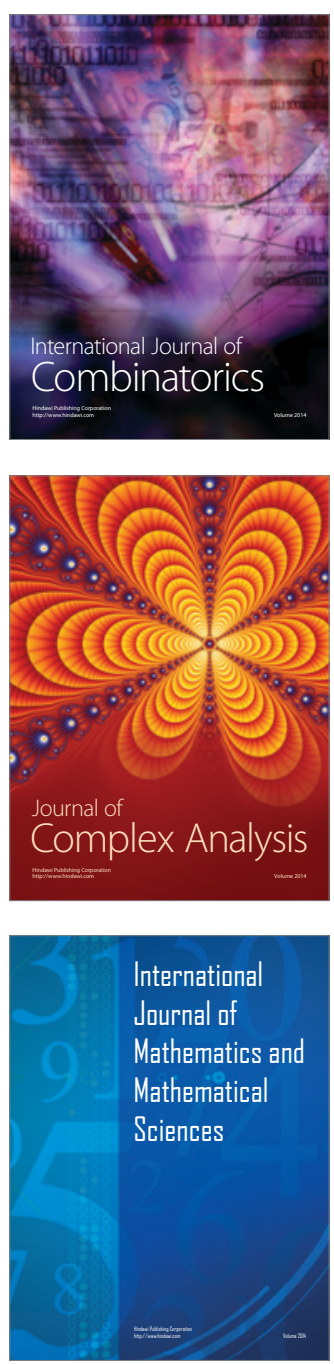
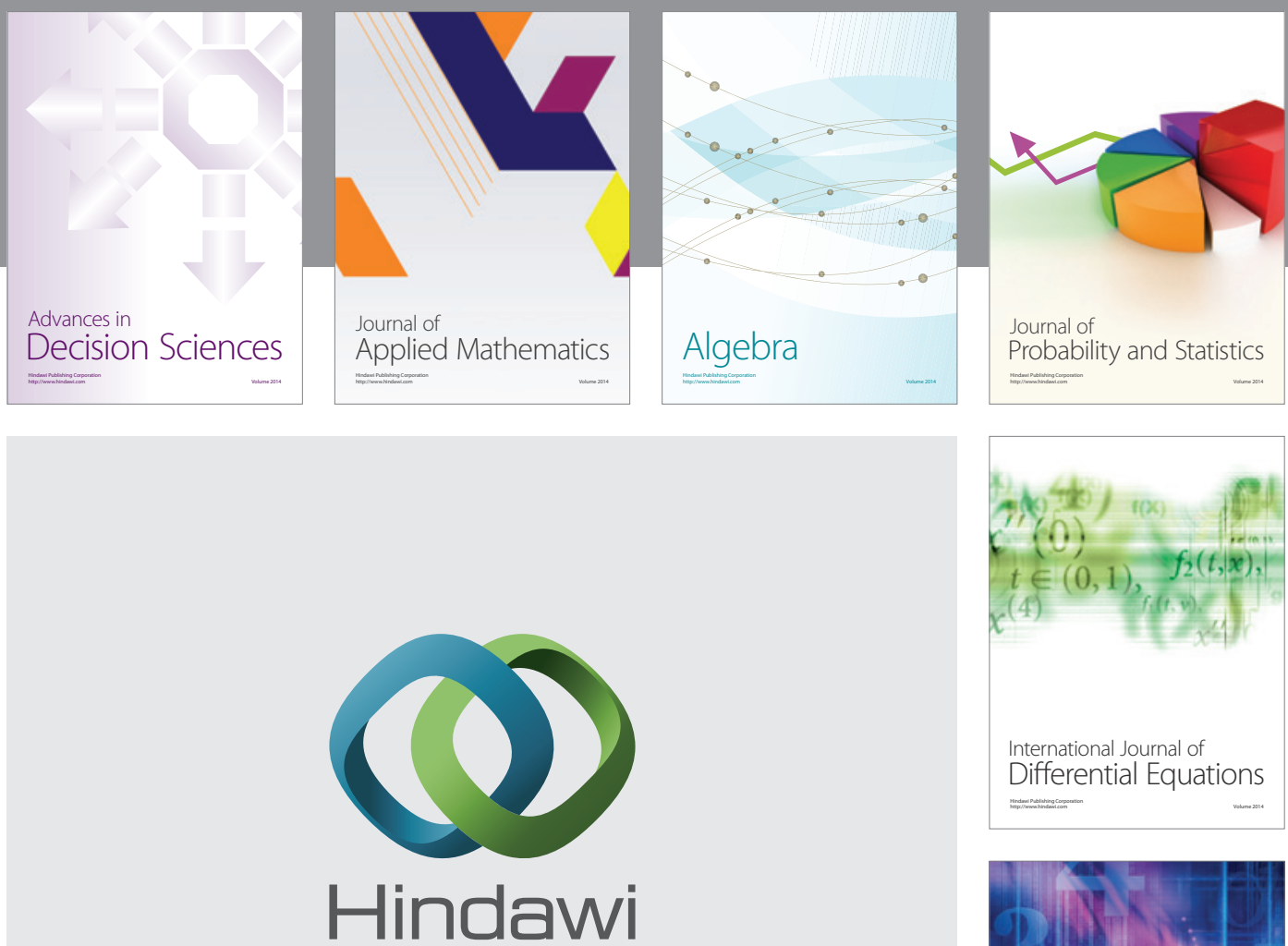

Submit your manuscripts at http://www.hindawi.com
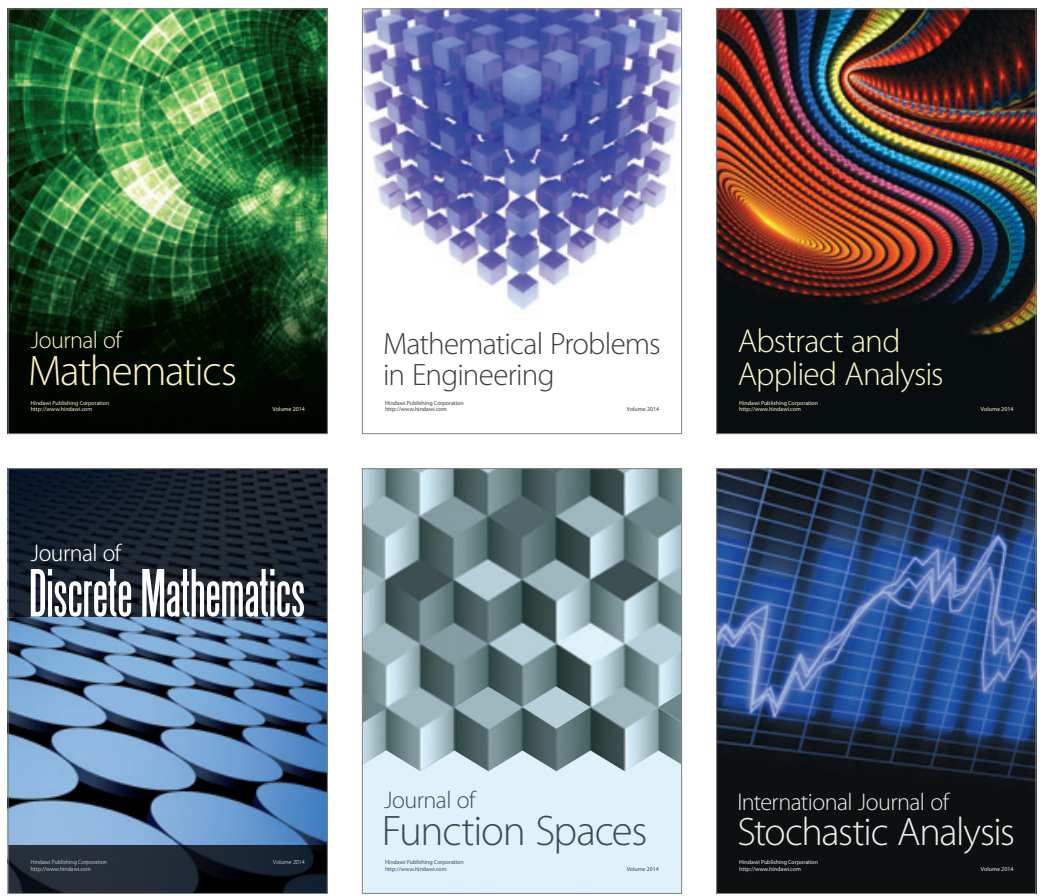

Journal of

Function Spaces

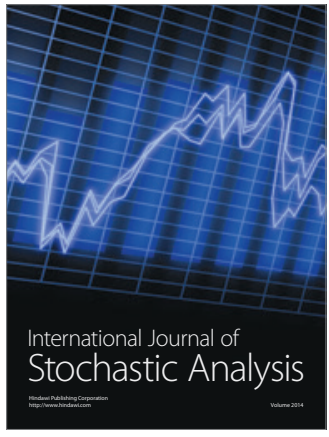

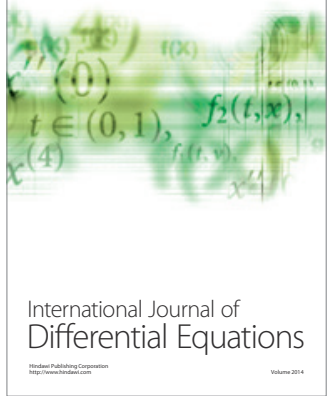
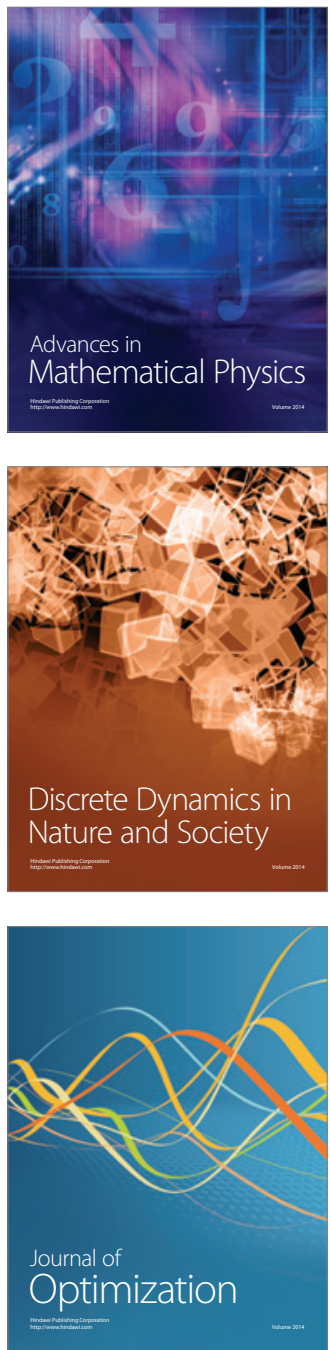